\title{
Communicable Diseases Report, NSW, March and April 2012
}

\author{
Communicable Diseases Branch \\ NSW Department of Health
}

For updated information, including data and facts on specific diseases, visit www.health.nsw.gov.au and click on Public Health and then Infectious Diseases. The communicable diseases site is available at: http://www.health.nsw.gov.au/publichealth/ infectious/index.asp.

Figure 1 and Tables 1 and 2 show notifications of communicable diseases received in March and April 2012 in New South Wales (NSW).

\section{Enteric infections \\ Outbreaks of suspected foodborne disease}

Ten outbreaks of gastrointestinal disease, thought to be due to the consumption of microbiologically contaminated food and which affected a total of 103 people, were reported in March and April 2012. This is higher than the number of outbreaks reported for the same period last year. These outbreaks were linked to restaurants $(n=6)$, takeaway shops $(n=2)$ and commercial caterers $(n=2)$. Of the 10 outbreaks; six were identified through complaints to the NSW Food Authority, three were reported directly to a public health unit, and one was detected through monitoring laboratory notifications of Salmonella clustered in time and space. Stool samples were tested in six of these outbreaks: Salmonella Typhimurium was found to be the cause in all of these.

There was insufficient data to draw conclusions about the likely cause for four outbreaks. In one of these outbreaks the cases had consumed a Bombe-Alaska from a Chinese restaurant. This dessert is covered with meringue made with raw egg and is known to be a high-risk food for salmonellosis because the meringue undergoes little or no cooking; any pathogens present in the egg therefore may cause illness. In three other outbreaks illness occurred in those who had consumed sandwich rolls and other items from Vietnamese bakeries or a crepe and kebab shop. Cross-contamination from raw ingredients is thought to be the cause of these outbreaks. Another outbreak occurred in people who ate bacon and egg burgers at a restaurant and the exact point of contamination of this well-cooked food could not be determined. In the final outbreak, illness was statistically significantly associated with eating a lamb salad however no pathogen, mechanism for contamination or bacterial growth or toxin could be identified.

\section{Outbreaks of gastroenteritis in institutional settings}

In March and April 2012, 108 outbreaks of gastroenteritis in institutions were reported, affecting 1805 people. This is $59 \%$ higher than for the same period last year (68 outbreaks). Thirty-seven outbreaks occurred in aged-care facilities, 61 in child-care centres, seven in hospitals, two in residential care units and one in a military facility. All of these outbreaks appear to have been caused by person-toperson spread of a viral illness. In $54(50 \%)$ outbreaks one or more stool specimens were collected. Norovirus was detected in the specimens from $23(43 \%)$ of these outbreaks. Rotavirus was detected in two (4\%) outbreaks. In $16(30 \%)$ outbreaks no pathogens were detected in stool specimens. Results for 13 outbreaks are outstanding.

Viral gastroenteritis increases in winter months. Public health units encourage institutions to submit stool specimens from case-patients for testing during an outbreak to help determine the cause of the outbreak (for further information see: Guidelines for the public health management of gastroenteritis outbreaks due to norovirus or suspected viral agents in Australia available at: http:// www.health.gov.au/internet/publications/publishing.nsf/ Content/cdacdna-norovirus.htm-1).

\section{Respiratory infections}

Influenza

Influenza activity in NSW, as measured by the number of people who presented with influenza-like illness to 59 of the state's largest emergency departments, was low during March and April 2012. In addition, laboratory surveillance identified only low numbers of influenza-positive specimens, although these were more than is usual for this time of year.

In March, there were:

- 106 presentations to emergency departments (rate 0.5 per 1000 presentations)

- 49 cases of laboratory-confirmed influenza including:

- 33 (67\%) influenza A

- $16(33 \%)$ influenza B. 
In April, there were:

- 110 presentations to emergency departments (rate 0.7 per 1000 presentations)

- 56 cases of laboratory-confirmed influenza including:

- $45(80 \%)$ influenza A

- $11(20 \%)$ influenza B.

For a more detailed report on respiratory activity in NSW see: http://www.health.nsw.gov.au/PublicHealth/Infectious/ influenza_reports.asp.

\section{Legionnaires' disease}

There were 19 cases of Legionnaires' disease reported in March and April 2012. Of these, 15 cases were due to Legionella pneumophila and two cases were due to Legionella longbeachae. Despite careful interviews with case-patients for common exposures and a review of the potential sources of infection (including cooling towers), no common environmental sources were identified for these cases.

Legionella bacteria can cause severe pneumonia if aerosolised water or dust that contains the bacteria is inhaled by susceptible people. Some air-conditioning cooling towers have been identified as the source of Legionnaires' disease outbreaks in the past as they can become contaminated by Legionella bacteria which are then aerosolised. There are requirements for building owners to register their cooling towers with local councils and to maintain cooling towers to minimise the growth of Legionella bacteria in the cooling tower water. For further information see: http:// www.health.nsw.gov.au/factsheets/environmental/legion_ control.html.

\section{Vaccine-preventable diseases Meningococcal disease}

Eleven cases of meningococcal disease were notified in NSW in March and April 2012 (three in March and eight in April); the age of the case-patients ranged from four months to 48 years and included five case-patients aged under 5 years. Eight cases were due to serogroup B (for which there is no vaccine), two cases were unable to be typed and one had missing information. There were no deaths notified in this period.

The number of cases is unchanged from the same period in 2011. Of the 11 cases notified in 2011 , six were due to serogroup B, one to serogroup W135, one to serogroup Y and for the remaining cases the serogroup was unknown. The ages of those affected ranged from 1 to 80 years, with four cases notified in children aged under 5 years.
It is recommended that a single dose of vaccine for meningococcal disease be given to all children at the age of 12 months as well as to those individuals at high risk of disease. $^{1}$

\section{Measles}

Three cases of measles were notified in NSW in March and April 2012. A 25 year-old man who acquired measles in Thailand infected his 9 month-old nephew and a soccer team contact after his return to Australia.

These are the first measles cases notified in 2012, following notifications in every month in 2011 . The number of cases has decreased from the same period in 2011, when there were 36 cases.

It is recommended that young adults travelling overseas should be up-to-date with their vaccinations, including that for measles.

\section{Sexually transmissible infections Gonorrhoea}

There has been an increase in the number of cases of gonorrhoea notified in NSW, with 970 reported in the first quarter of 2012 compared to 608 in the same period in 2011. The increase in gonorrhoea notifications has been noted across most local health districts and in both men and women. The highest risk group continues to be men aged 25-44 years (who account for nearly $50 \%$ of all notifications).

Part of the increase in the reporting of gonorrhoea may be due to more testing and better laboratory diagnoses. Campaigns have aimed to increase testing rates for sexually transmissible infections in those at highest risk. A number of laboratories have also recently introduced new testing strategies which may be resulting in more cases of gonorrhoea being identified.

Gonorrhoea is a type of bacteria that can infect the urethra (the tube that carries urine from the bladder to outside), throat and anus in both men and women and the cervix (neck of the womb) in women. It can be effectively treated by a single dose of antibiotics. Using a condom for vaginal or anal sex can significantly reduce the risk of catching gonorrhoea and other sexually transmissible infections.

\section{References}

1. National Health and Medical Research Council. The Australian Immunisation Handbook. 9th ed. Canberra: Australian Government Department of Health and Ageing; 2008. 
Figure 1. Reports of selected communicable diseases, NSW, January 2004 to April 2012, by month of onset.

Preliminary data: case counts in recent months may increase because of reporting delays.

Laboratory-confirmed cases only, except for measles, meningococcal disease and pertussis.

$\mathrm{BFV}=$ Barmah Forest virus infections, RRV $=$ Ross River virus infections,

lab conf = laboratory confirmed,

Men $\mathrm{Gp} C$ and $\mathrm{Gp} \mathrm{B}=$ meningococcal disease due to serogroup $\mathrm{C}$ and serogroup $\mathrm{B}$ infection,

other/unk $=$ other or unknown serogroups.

NB: Multiple series in graphs are stacked, except gastroenteritis outbreaks.

NB: Outbreaks are more likely to be reported by nursing homes and hospitals than by other institutions.

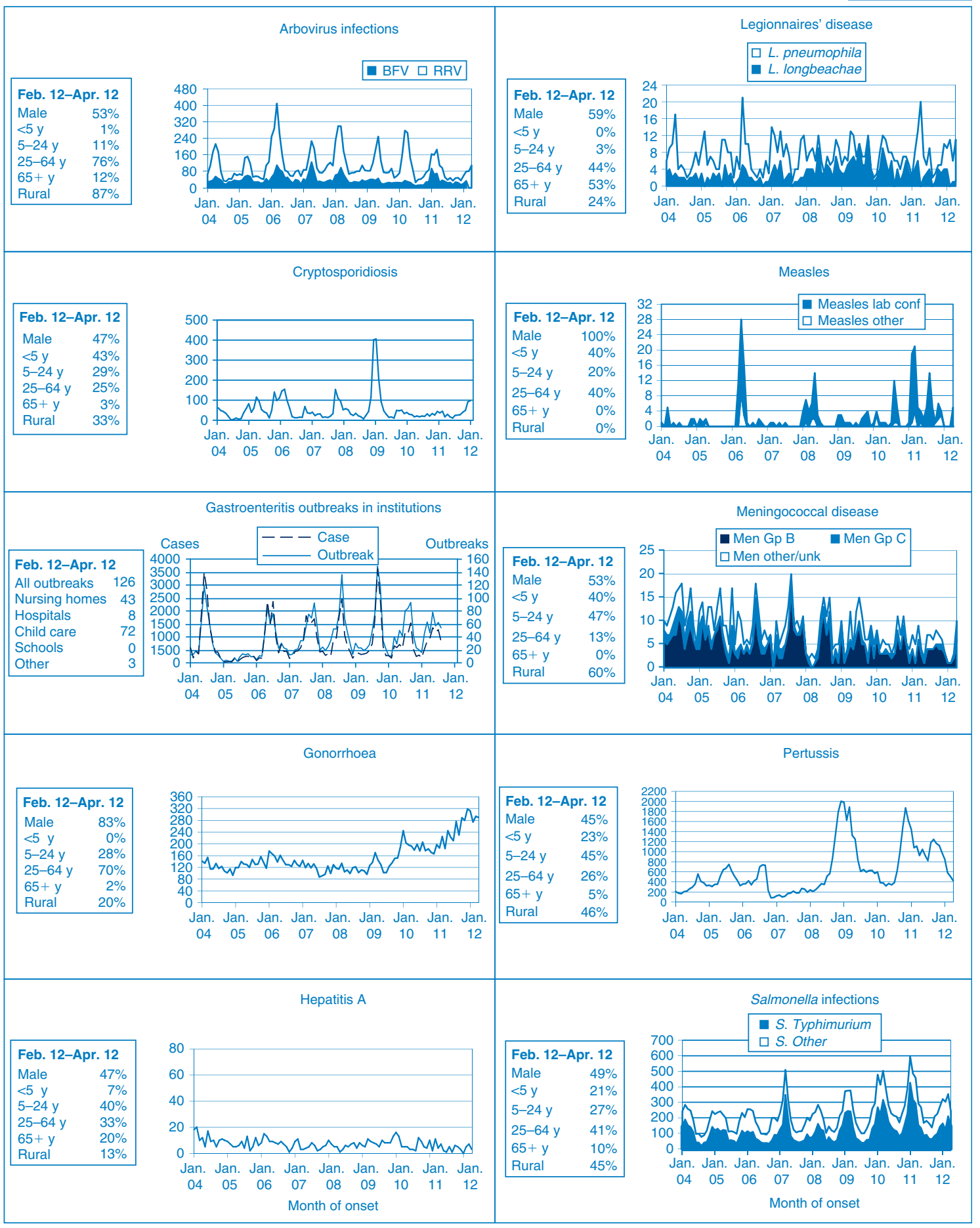




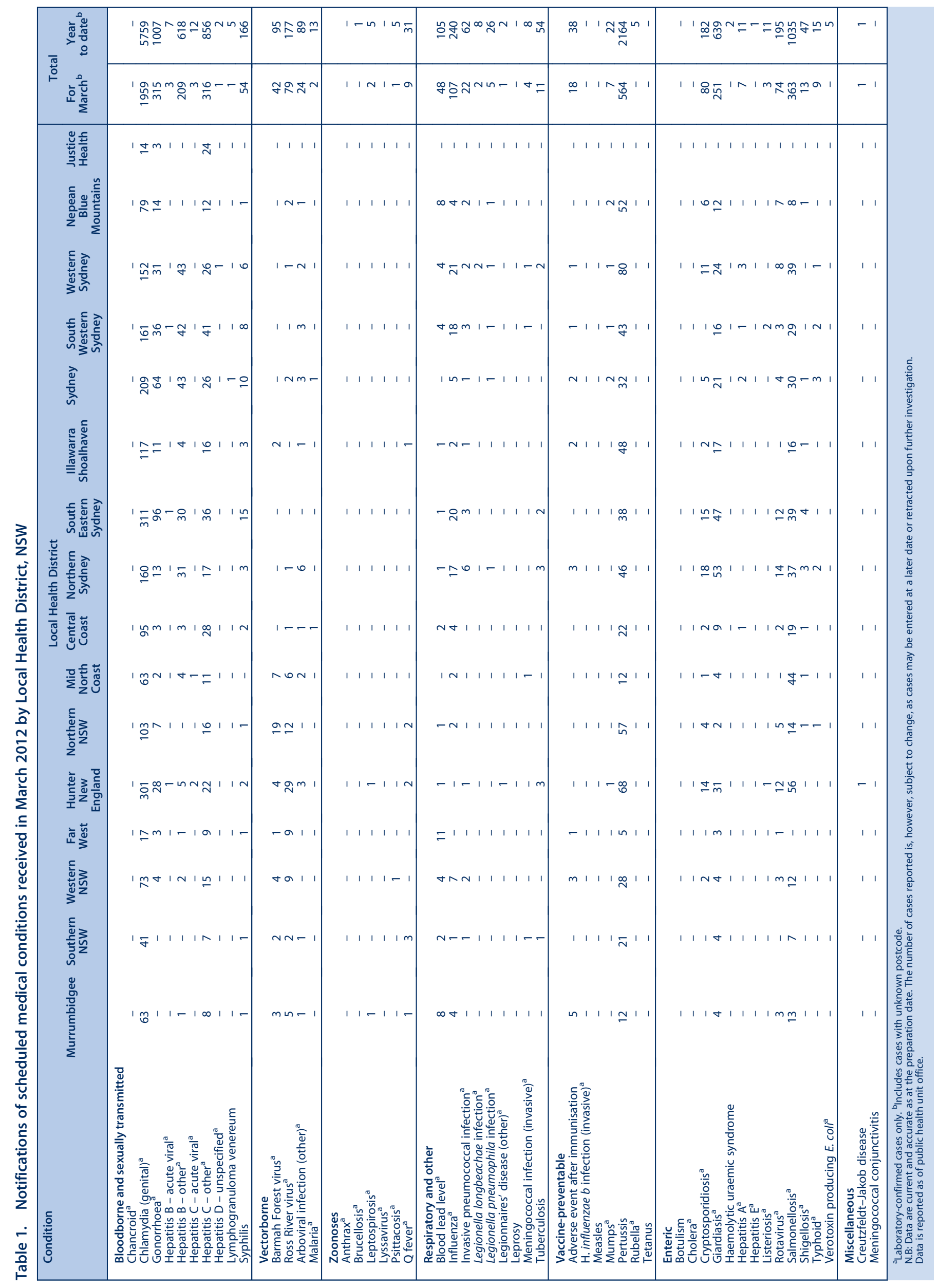




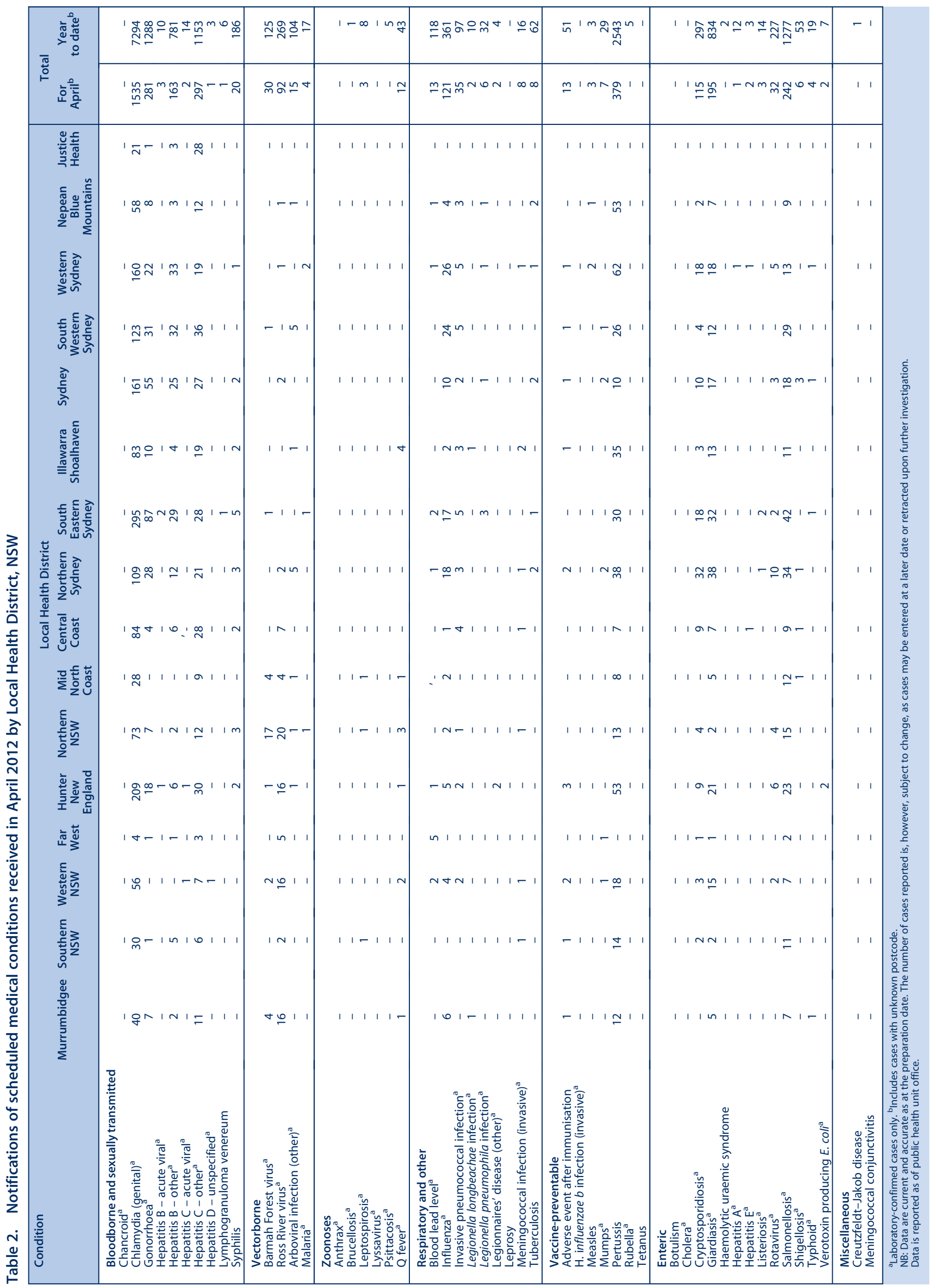

